\title{
Strategies for In-Person Recruitment: Lessons Learned from a New Jersey Primary Care Research Network (NJPCRN) Study
}

\author{
Christina B. Felsen, MPH, Eric K. Shaw, PhD, Jeanne M. Ferrante, MD, MPH, \\ Lorraine J. Lacroix, MPH, and Benjamin F. Crabtree, $\mathrm{PhD}$
}

Objective: To describe and evaluate participant recruitment for a research study conducted in primary care offices.

Methods: Nine recruiters administered a written survey to 1485 primary care patients (from 25 practices) during baseline and 1-year follow-up of a quality improvement study aimed at increasing colorectal cancer screening. Before recruitment, recruiters attended training sessions, during which they received tools and information designed to facilitate successful recruitment. Quantitative and qualitative recruitment data were analyzed to assess and describe recruitment efforts.

Results: The overall practice-level recruitment rate was $72.7 \%$ (range, $56.3 \%$ to $91.4 \%$ ). Practice characteristics did not affect the recruitment rate. Recruitment rate differed significantly between recruiters $(P=.0007)$ as did nonparticipants' reasons for refusal $(P<.0001)$. Anticipated barriers to recruitment (older age of sampled population, lack of incentives, and discomfort discussing colorectal cancer) did not occur. Two key strategies facilitated recruitment: (1) recruiter flexibility and (2) building rapport with participants.

Conclusion: Recruiters may be more effective if they are able to adapt to participants' needs and successfully build rapport with potential participants. The likelihood of recruitment success may be increased by anticipating potential recruitment barriers and providing training that minimizes the inherent variation that exists among recruiters. (J Am Board Fam Med 2010;23:523-533.)

Keywords: Cancer Research, Participant Recruitment, Primary Health Care

Adequate and timely participant recruitment can determine a research study's success. Enrolling a targeted sample size during a specified time period helps ensure sufficient statistical power ${ }^{1,2}$ and keeps study operations within budget, time, and person-

This article was externally peer reviewed.

Submitted 27 April 2009; revised 31 August 2009; accepted 8 September 2009.

From the Department of Family Medicine (CBF, EKS, JMF, BFC) and The Cancer Institute of New Jersey (JMF, BFC), University of Medicine and Dentistry of New JerseyRobert Wood Johnson Medical School, New Brunswick; the Department of Family Medicine, University of Medicine and Dentistry of New Jersey-New Jersey Medical School, Newark (JMF); and the College of Nursing, Rutgers University, Newark, NJ (LJL).

Funding: This manuscript comprises data arising from the Supporting Colorectal Outcomes through Participatory Enhancements study, which is funded by the National Cancer Institute (grant no. 1R01CA112387-01).

Conflict of interest: none declared.

Corresponding author: Christina B. Felsen, MPH, Department of Family Medicine, Research Division, University of Medicine and Dentistry of New Jersey-Robert Wood Johnson Medical School, 1 World's Fair Drive, Somerset, NJ 08873 (E-mail: felsencb@umdnj.edu). nel constraints. ${ }^{3,4}$ In-person recruitment of participants is one technique that has been shown to be both successful ${ }^{5-8}$ and cost-effective ${ }^{9-11}$ in multiple study types; however, researchers rarely report the strategies used to facilitate successful in-person recruitment.

Several attributes of successful recruiters have been identified, including flexibility in recruitment techniques ${ }^{5,7,11}$ and dedication to the research. ${ }^{11,12}$ In addition, study participants identify competent, personable, and experienced recruiters as a positive influence on their decision to participate in research. ${ }^{8,13}$ Although these findings point to the importance of adequately training recruiters, very little documentation exists about the actual training methods researchers use to do this. ${ }^{14,15}$

This article describes participant recruitment for Project SCOPE (Supporting Colorectal Cancer Outcomes through Participatory Enhancements). Barriers to recruitment success were anticipated based on the older age of the sampled population, 
the sensitive nature of colorectal cancer (CRC), and the lack of incentives. In this article we report on recruitment outcomes and offer strategies to aid other researchers who are conducting in-person recruitment. Qualitative examples of recruiters' experiences are used to provide concrete and practical information that is often left out of scholarly work pertaining to participant recruitment.

\section{Methods}

\section{Description of SCOPE Study}

Project SCOPE was a 5-year quality improvement intervention study funded by the National Cancer Institute. SCOPE used a multiple-method assessment process ${ }^{16}$ to inform a facilitated, team-building intervention ${ }^{17}$ that aimed to increase CRC screening rates in primary care practices. A convenience sample of 25 family and internal medicine practices were recruited from the New Jersey Primary Care Research Network. Each participating practice provided written, informed consent, as did all participants. The University of Medicine and Dentistry of New Jersey-Robert Wood Johnson Medical School Institutional Review Board approved this study.

\section{Data Collection}

Baseline and 1-year follow-up data were collected during January 2006 through May 2008. Data were collected in 25 practices at baseline and in 23 practices at 1-year follow-up ( 2 practices closed during the follow-up period). Recruiters approached consecutive patients in the waiting rooms of each practice in an effort to recruit 30 participants who consented to completing a survey and having their medical record reviewed. Occasionally, 1 to 2 additional participants were recruited to account for drop outs or missing records. Eligible patients were 50 years of age or older, had visited the practice at least once before, and could read/write in English or Spanish. Participants completed a written survey that included demographics, risk factors, cancer screening dates, health care-seeking behaviors, self-rated health, and satisfaction with care. Incentives were not provided to participants.

\section{Participant Recruitment}

Nine recruiters enrolled participants during the course of the study: 3 during baseline and 6 during 1-year follow-up. All but one of these recruiters were women and most fell into the 20 - to 40 -yearold age range. The educational backgrounds of the recruiters varied and included high school graduates, MPH students and graduates, a registered nurse, and a $\mathrm{PhD}$ in sociology. The racial backgrounds of recruiters also varied and included white, Hispanic, Asian Indian, and African American. Only one recruiter had previous experience with enrolling research study participants, but others had related experience, including working in a primary care setting and approaching individuals for market research purposes. Two of the recruiters were bilingual and were responsible for recruitment at practices with large Spanish-speaking patient populations. Monolingual recruiters worked with English-speaking participants only.

Recruiters followed a standard recruitment protocol designed by the research team (Figure 1). The length of time recruiters spent at each practice ranged from 3 to 18 days and varied based on the practice's schedule and patient volume.

The following details a typical interaction between a recruiter and a patient:

Context: Hilltop practice has a small waiting room with approximately 15 chairs. An elderly woman enters the practice and approaches the front desk window. The receptionist speaks with the patient and then asks her to have a seat. The recruiter gathers her clipboard, survey, and pen and approaches the patient, whose age is unknown.

Recruiter: Good morning, may I interrupt you?

Patient: Sure.

Recruiter: My name is Emma and I'm from the University of Medicine and Dentistry of New Jersey [pointing to her identification badge]. We are currently working with your practice to help improve cancer screening rates. To do this, we are asking patients who have been seen at this office before and who are at least 50 years old if they'd be willing to fill out a brief survey. Are you at least 50 years old?

Patient: Yes.

Recruiter: Have you been seen by Dr. Roberts before?

Patient: Yes, I've been coming here for years.

Recruiter: That means you would be eligible to complete the survey if you're interested. It takes about 15 minutes so you can complete it while you wait. We keep all information confidential and won't contact you in the future. After you complete the survey, a nurse that I work with will come in and review parts of your medical record that per- 
Figure 1. Participant recruitment protocol for Supporting Colorectal Outcomes through Participatory Enhancements (SCOPE).

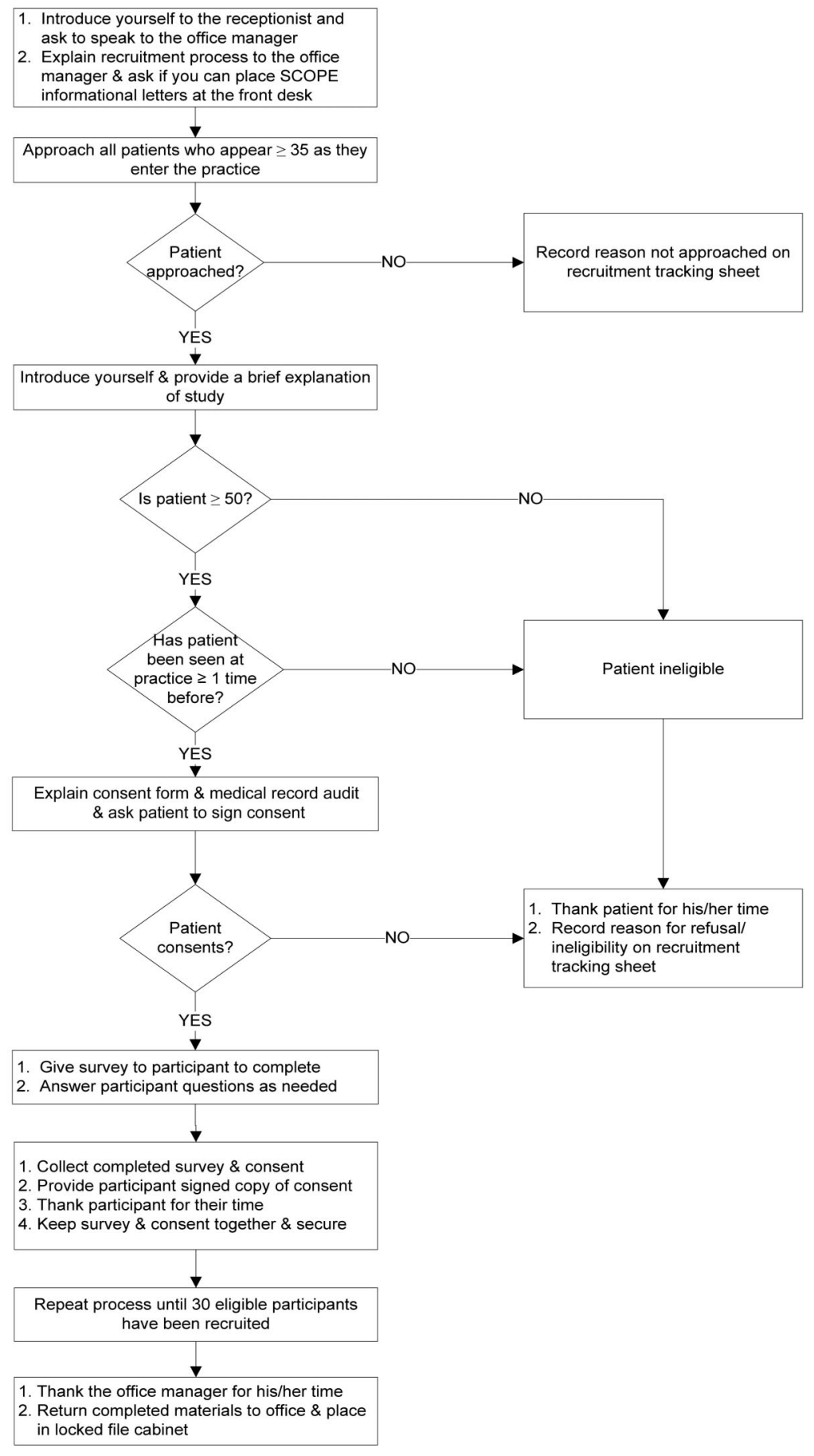

tain to cancer screening. Would you be willing to fill out the survey today?

If the patient consented, the recruiter would then review the consent form and assist with the survey if needed. If the patient refused, recruiters would respond positively (eg, "Thank you anyway." or "Have a wonderful day!") and would then ask for and record the patient's reason for refusal. Recruiters sometimes failed to approach potentially eligible participants because the patient was called into the examination room immediately or because the recruiter was busy assisting another participant. 


\section{Analysis of Recruitment Data}

Recruitment rates represent the percentage of patients that were approached who consented to participate. Analyses of variance were used to evaluate the effect of recruiter and practice characteristics on recruitment rates. A $\chi^{2}$ test was conducted to evaluate the difference in nonparticipants' refusal reasons between recruiters. Qualitative data about recruiters' experiences were collected during an exploratory debriefing session that included 4 recruiters who were working full-time on the project at the time of the session. During the session, recruiters discussed the challenges they faced during recruitment and techniques used to facilitate recruitment, and notes were taken by one of the authors $(\mathrm{CBF})$.

\section{Data Quality}

\section{Recruiter Training}

Before beginning recruitment, recruiters attended a day-long, in-house training session and also shadowed more experienced recruiters. Descriptions of the training modules are provided below:

1. Project Overview. A history of SCOPE's development and an overview of the project's structure, timeline, and primary goals were given. This presentation provided context for the recruiters and prepared them to knowledgeably answer questions from patients and practice members. National and local CRC mortality and screening statistics were presented to provide background about the study's significance.

2. Data Collection. Experienced recruiters reviewed the participant survey with the new recruiters and explained how to answer questions commonly raised by participants. Articles that defined the CRC screening tests and other medical terms used in the survey ${ }^{18,19}$ were discussed, as were strategies for tactfully explaining these tests to participants. A document covering frequently asked questions about recruitment and appropriate responses (developed based on earlier experiences) was also discussed (Appendix 1). Finally, tips for being an effective recruiter were presented, including the importance of a professional appearance and friendly demeanor.

3. Patient Consent. Experienced recruiters reviewed methods for obtaining informed consent and the key points of the consent form, including (1) participation is voluntary, (2) there are no foreseen risks to participation, (3) participants have the right to withdraw at any time, and (4) all participant information is kept confidential. New recruiters were instructed that family members or caretakers could not provide proxy consent for participants. Making sure each participant understood that providing consent included agreeing to both the survey and a one-time audit of their medical record was also emphasized.

4. Confidentiality. This module covered the importance of participant confidentiality and prepared recruiters to evaluate and respond to situations in which confidentiality might be compromised. New recruiters were instructed to keep completed surveys secure and maintain possession of completed materials at all times (eg, when taking a break or leaving the practice for lunch). Situations requiring recruiters to judge whether privacy could be maintained were discussed, including introducing the study to a patient with a hearing impairment or reading the survey to a visually impaired patient in a crowded waiting room. New recruiters were given examples of the appropriate way to respond in these situations and were instructed to always maintain participant privacy, even if that meant refusing an individual that was otherwise willing to participate.

5. Simulated Recruitment Exercises. New recruiters participated in role-playing exercises to become more comfortable with approaching patients. Experienced recruiters provided a demonstration of a typical patient interaction that focused on assessing eligibility, giving a brief introduction of the study, and obtaining written consent. Although a standardized script was used in this demonstration, recruiters were encouraged to adapt the script to different situations if necessary. To do this, recruiters participated in role-playing scenarios that involved unexpected situations, such as encountering individuals who were very emotional because of a recent cancer diagnosis or were offended that the recruiter assumed they were older than 50 . Recruiters were challenged to quickly think of ways to respond appropriately and professionally, and were given on-the-spot, constructive feedback. 
6. Shadowing. In addition to the in-house training, new recruiters also spent 2 to 3 days observing an experienced recruiter in real-life interactions with patients. During this time, the new recruiters approached patients on their own while under the supervision of the experienced recruiter. This proved to be an especially important part of the training because it helped new recruiters gain confidence in approaching patients and it provided learning opportunities to improve their recruitment techniques.

\section{Continuous Quality Control}

Although recruiters worked independently in the practices, project management took several steps to monitor recruitment and help recruiters problemsolve while in the field. Recruiters were instructed to call if there was a question they could not answer or if they faced a situation in which they were uncertain how to proceed. This helped ensure that recruiters followed the standardized recruitment protocol, even when faced with unique challenges. Project management also met regularly with recruiters to give them opportunities to discuss issues arising from their work in the field and to share their experiences. These meetings helped minimize recruiter isolation and ensured that situations were being handled similarly by all recruiters.

\section{Results \\ Practice Characteristics and Overall Recruitment Outcomes}

Most practices were physician-owned family medicine practices located in suburban areas. More than half of the patients at $72 \%$ of the practices were privately insured and only one practice served a patient population that was $>75 \%$ uninsured. The recruitment rate ranged from $56.3 \%$ to $91.4 \%$ among practices (median, 75.6; Q1, 70.0; Q3, 79.5). There was no association between practice characteristics and recruitment rates (Table 1).

\section{Participant Characteristics}

A total of 2498 patients were approached during baseline and 1-year follow-up. Two thousand fortytwo patients were eligible to participate and of these, 1485 (72.7\%) consented to participate (Figure 2). Participant characteristics are described in Table 2. Most participants were white, female, and married with at least a high school education (Table 2).

\section{Recruitment Outcomes between Recruiters}

Average recruitment rates differed significantly among recruiters $(\mathrm{F}=4.44 ; P=.0007)$. Overall, the main reasons patients refused to participate were lack of interest in the survey/study $(28.1 \%)$ or objection to the medical record review, signing the consent form, or completing the survey $(21.0 \%)$. Other common refusal reasons included being too busy/not having enough time (15.6\%) and being sick/disabled (17.2\%). None of the nonparticipants stated that they were uncomfortable discussing CRC or that they refused because an incentive was not offered. Nonparticipants' reasons for refusal differed significantly among recruiters $\left(\chi^{2}=133.5\right.$; $P<.0001)$; certain recruiters were more likely to encounter patients that objected to the survey, chart audit, or consent form or were not interested in the study (Table 3).

\section{Strategies for Recruitment Success}

During the debriefing session, recruiters identified several challenges common to their individual recruitment experiences. These included interacting with ill patients and finding alternate ways to explain the study to those who had trouble understanding. From these challenges, recruiters identified 2 key strategies that facilitated recruitment success: (1) flexibility and (2) building rapport.

\section{Recruiter Flexibility}

Recruiters often found it necessary to adapt the protocol to accommodate individual participants' needs. For example, some patients were eager to participate but needed recruiters to read the questions to them because they had trouble seeing. In such cases, recruiters would attempt to take the individual to a previously identified private area (eg, an empty examination room). If this area was not available, recruiters would determine whether it was feasible to assist the patient without compromising confidentiality. If privacy could not be guaranteed then the recruiter explained to the patient that they would not be able to participate and thanked them for their time.

Recruiters also adapted their approach to patients based on the patient's body language and/or temperament. For example, if a patient expressed anger or that they were in a hurry while interacting 
Table 1. Characteristics of Practices in Supporting Colorectal Outcomes through Participatory Enhancements (SCOPE), New Jersey, 2006-2008

\begin{tabular}{|c|c|c|c|}
\hline Practice Characteristics & $\mathrm{n}(\%)$ & Average Recruitment Rate (\%)* & $P$ \\
\hline Total sample & $25(100)$ & & \\
\hline \multicolumn{4}{|l|}{ Type of practice } \\
\hline Family medicine & $18(72.0)$ & 75.5 & \\
\hline Internal medicine & $5(20.0)$ & 79.2 & \\
\hline Family and internal medicine & $2(8.0)$ & 73.7 & .7006 \\
\hline \multicolumn{4}{|l|}{ Physicians per practice } \\
\hline 1 & $6(24.0)$ & 79.8 & \\
\hline $2-5$ & $12(48.0)$ & 74.6 & \\
\hline$>5$ & $7(28.0)$ & 75.2 & .5275 \\
\hline \multicolumn{4}{|l|}{ Mid-level providers } \\
\hline None & $13(52.0)$ & 75.0 & \\
\hline Nurse practitioners & $5(20.0)$ & 80.4 & \\
\hline Physician assistants & $4(16.0)$ & 79.9 & \\
\hline Both nurse practitioner and physician assistant & $3(12.0)$ & 67.0 & .3483 \\
\hline \multicolumn{4}{|l|}{ Ownership type } \\
\hline Physician & $19(76.0)$ & 75.4 & \\
\hline Hospital health system & $3(12.0)$ & 69.0 & \\
\hline University & $2(8.0)$ & 90.5 & \\
\hline Other & $1(4.0)$ & 76.7 & .0980 \\
\hline \multicolumn{4}{|l|}{ Insurance type of patients seen at practice (\%) } \\
\hline \multicolumn{4}{|l|}{ Private } \\
\hline$<50$ & $7(28.0)$ & 75.1 & \\
\hline $50-75$ & $12(48.0)$ & 76.5 & \\
\hline$>75$ & $6(24.0)$ & 75.8 & .9549 \\
\hline \multicolumn{4}{|l|}{ Medicare } \\
\hline$<50$ & $23(92.0)$ & 75.9 & \\
\hline $50-75$ & $2(8.0)$ & 77.0 & .8951 \\
\hline \multicolumn{4}{|l|}{ Medicaid } \\
\hline$<50$ & $23(92.0)$ & 76.7 & \\
\hline $50-75$ & $2(8.0)$ & 67.0 & .1653 \\
\hline \multicolumn{4}{|l|}{ None } \\
\hline$<50$ & $23(92.0)$ & 75.4 & \\
\hline $50-75$ & $1(4.0)$ & 87.6 & \\
\hline$>75$ & $1(4.0)$ & 76.7 & .4672 \\
\hline \multicolumn{4}{|l|}{ Location } \\
\hline Urban & $4(16.0)$ & 81.9 & \\
\hline Suburban & $20(80.0)$ & 74.9 & \\
\hline Rural & $1(4.0)$ & 78.9 & .3674 \\
\hline
\end{tabular}

*Average of recruitment rates at baseline and 1-year follow-up.

with the office staff, the recruiter's introduction would remain friendly but be quicker and more to the point. If a patient complained of not feeling well or was visibly sick, a recruiter might adopt a sympathetic tone of voice when speaking to the patient and acknowledge their illness by saying, "I can see you're not feeling well, but do you mind if I have a few moments of your time?" These small adaptations in the way recruiters approached pa- tients helped put patients at ease and seemed to encourage them to consider participation.

Recruiters also had to adapt the recruitment script when they sensed that a potential participant did not understand the presented information. Many participants were quick to agree to the survey, but became confused about what participation entailed once they had read the consent form. Recruiters often had to explain the complicated con- 
Figure 2. Summary of participant recruitment results in Supporting Colorectal Outcomes through Participatory Enhancements (SCOPE), New Jersey, 2006-2008.

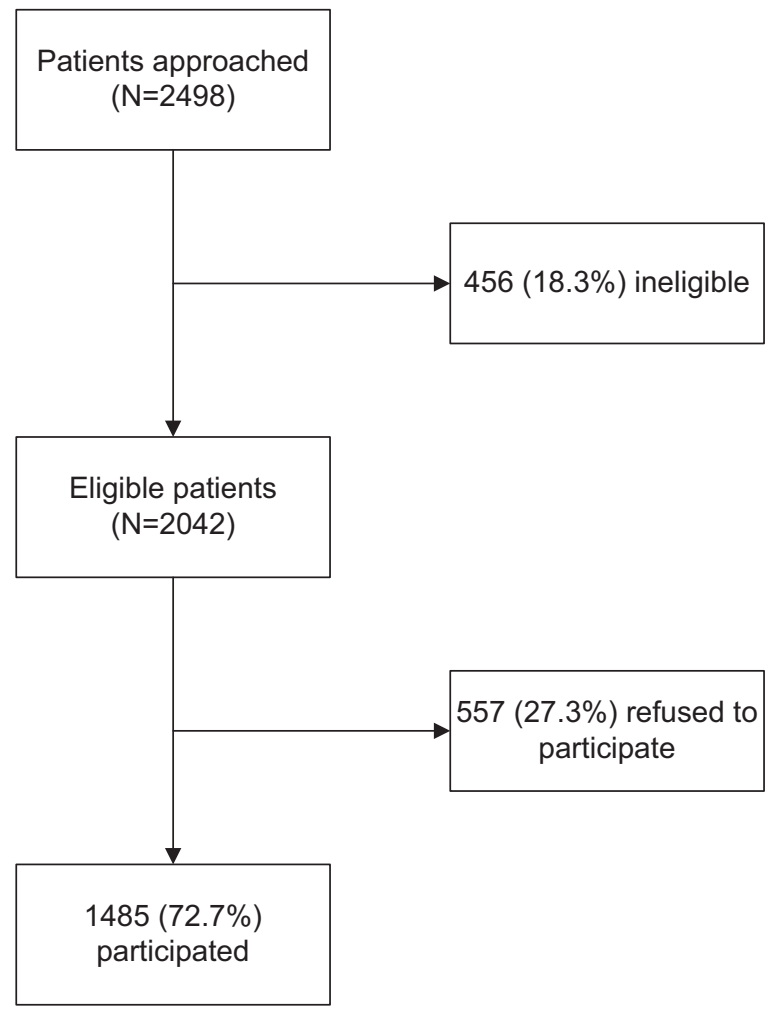

sent form, provide a more detailed explanation of the study's purpose, and assure the participant that their participation was limited to a one-time survey and medical record review only. Recruiters found that spending extra time with hesitant participants helped to alleviate concerns and increase their acceptance of the study.

\section{Building Rapport}

Recruiters recognized that patients may have viewed being asked to participate as a nuisance, especially if they were sick, in a hurry, or did not fully understand the study's purpose. Acknowledging these circumstances with an understanding statement, a concerned look, or the provision of more information often helped to build rapport with potential participants. Small talk proved to be an effective way to engage some individuals who initiated conversations about topics unrelated to the study such as their family, job, or current events. Participating in these conversations helped recruiters gain participants' trust and confidence.
Table 2. Characteristics of Participants in Supporting Colorectal Outcomes through Participatory

Enhancements (SCOPE), New Jersey, 2006-2008

\begin{tabular}{lc}
\hline Participant Characteristics & $\mathrm{n}(\%)$ \\
\hline Total sample & $1485(100)$ \\
Age (years) & \\
$50-59$ & $592(39.9)$ \\
$60-69$ & $481(32.4)$ \\
$\geq 70$ & $412(27.7)$ \\
Sex & \\
Female & $882(59.4)$ \\
Male & $603(40.6)$ \\
Race* & \\
White & $1074(72.8)$ \\
Black & $232(15.7)$ \\
Hispanic & $102(6.9)$ \\
Other & $68(4.6)$ \\
Marital status* & \\
Married & $932(63.0)$ \\
Not married & $546(37.0)$ \\
Education level* & \\
$<$ High school & $166(11.3)$ \\
High school diploma or some college & $723(49.0)$ \\
College or graduate school degree & $586(39.7)$ \\
Insurance* & \\
Private & $718(49.0)$ \\
Medicare & $553(37.7)$ \\
Medicaid & $77(5.3)$ \\
Other & $56(3.8)$ \\
None & \\
\hline
\end{tabular}

*Numbers do not add to total because of missing data.

Participants sometimes needed reassurance that recruiters were nearby if they had questions, and recruiters found that sitting near these participants and emphasizing that they were available to assist helped put them at ease. Other participants asked recruiters for additional assistance, such as entertaining their grandchildren in the waiting room so they were free to participate. Being able to accommodate a wide range of circumstances was therefore an essential skill for recruiters to possess.

\section{Discussion}

This analysis shows that comprehensive training of recruiters and effective project management (eg, ongoing skill-building opportunities for recruiters and consistent monitoring of recruitment efforts) are key to achieving a high in-person recruitment rate in research studies. Diverse training meth- 
Table 3. Recruitment Rates and Nonparticipant Refusals among Recruiters in Supporting Colorectal Outcomes through Participatory Enhancements (SCOPE), New Jersey, 2006-2008

\begin{tabular}{|c|c|c|c|c|c|c|c|c|}
\hline \multirow[b]{2}{*}{ Recruiter } & \multirow[b]{2}{*}{$\begin{array}{l}\text { Practices } \\
\text { (n) }\end{array}$} & \multirow[b]{2}{*}{$\begin{array}{c}\text { Refusals } \\
\text { (n) }\end{array}$} & \multirow[b]{2}{*}{$\begin{array}{c}\text { Average } \\
\text { Recruitment } \\
\text { Rate (\%) }\end{array}$} & \multicolumn{5}{|c|}{ Reason for Refusal (n [\%]) } \\
\hline & & & & Not Interested & $\begin{array}{c}\text { No } \\
\text { Time/ } \\
\text { Too Busy }\end{array}$ & $\begin{array}{c}\text { Uncomfortable with } \\
\text { Chart Audit, } \\
\text { Survey, Consent }\end{array}$ & $\begin{array}{l}\text { Illness/ } \\
\text { Disability }\end{array}$ & Other \\
\hline 1 & 22 & 181 & 81.7 & $42(26.75)$ & $26(16.56)$ & $50(31.85)$ & $27(17.20)$ & $12(7.64)$ \\
\hline 2 & 7 & 98 & 68.1 & $58(61.70)$ & $9(9.57)$ & $7(7.45)$ & $12(12.77)$ & $8(8.51)$ \\
\hline 3 & 5 & 67 & 71.9 & $9(14.75)$ & $19(31.15)$ & $7(11.48)$ & $12(19.67)$ & $14(22.95)$ \\
\hline 4 & 2 & 43 & 61.3 & $6(14.63)$ & $3(7.32)$ & $11(26.83)$ & $6(14.63)$ & $15(36.59)$ \\
\hline 5 & 5 & 122 & 57.0 & $25(20.83)$ & $17(14.17)$ & $21(17.50)$ & $19(15.83)$ & $38(31.67)$ \\
\hline 6 & 3 & 49 & 68.1 & $14(32.56)$ & $2(4.65)$ & $9(20.93)$ & $11(25.58)$ & $7(16.28)$ \\
\hline $7,8,9^{*}$ & 3 & 43 & 71.4 & $3(7.32)$ & $11(26.83)$ & $12(29.27)$ & $9(21.95)$ & $6(14.63)$ \\
\hline
\end{tabular}

*Recruiters 7, 8, and 9 each recruited at one practice only and are therefore collapsed into one category for the purposes of this analysis.

ods-including didactic presentations, role-playing exercises, and shadowing of experienced personnel—should be used to interest and invest recruiters in the study and prepare them to knowledgably address participant questions and unexpected situations. Through the SCOPE recruiter training, as well as the regular meetings with project management, recruiters became motivated, confident, and proficient at identifying strategies to facilitate recruitment and executing strategies to overcome potential barriers to recruitment.

Two strategies in particular were important to our recruitment success: flexibility and building rapport with participants. Although flexibility has previously been identified as an attribute of successful recruitment, ${ }^{5,7,11}$ this article contains one of the few published accounts of the training methods used to help in-person recruiters develop adaptive techniques without violating study protocol. We also present examples of the human factors associated with in-person recruitment (eg, ill or emotional participants) that challenge recruiters to find creative ways to build rapport in a wide range of participant interactions. We found that having regular opportunities for recruiters to discuss their experiences led to the development of shared strategies for being flexible and building rapport.

Several barriers to recruitment were anticipated based on the older age of the participant population, ${ }^{5,12}$ participants' potential embarrassment discussing CRC screening tests, ${ }^{20,21}$ and the lack of incentives. ${ }^{11}$ Our high recruitment rate suggests that the older age of the sampled population did not adversely affect recruitment. Recruiters actu- ally found elderly patients (ie, participants in their 70 s and 80 s) to be more receptive and talkative than other participants. Surprisingly, none of the nonparticipants stated they were uncomfortable discussing CRC, and recruiters found that most participants did not express embarrassment, even when recruiters explicitly defined the invasive screening tests. The lack of incentives was never given as a reason for refusal, although some patients occasionally asked "what's in it for me?" Anticipating barriers in advance and providing ongoing training through regular meetings can help recruiters identify ways to overcome recruitment challenges and may help eliminate some of the common pitfalls of participant recruitment.

No matter how skilled or experienced recruiters are, variability is to be expected when a single recruiter enrolls participants at multiple sites., We anticipated that individual recruiter rates would vary slightly across practices as the recruiter gained expertise in and confidence with recruiting. However, we did not expect the large degree of variability that existed in some recruiter's rates. For example, recruiter 1 conducted recruitment at 22 of the 25 practices at baseline, producing recruitment rates that ranged from $61.5 \%$ to $97.0 \%$. This large range suggests that practice factors not available for this analysis affected recruitment outcomes.

Despite our efforts to standardize the recruitment process, variability between recruiters also had an impact on recruitment outcomes. For example, recruiters 1 and 5 encountered a greater number of patients who refused because they were uncomfortable with the survey, chart audit, or con- 
sent. Possible explanations for this include a lack of clarity in these recruiters' explanations of participation or a difference in the way the recruiters recorded the nonparticipants' refusal reasons. Variability between recruiters may also be explained by patient characteristics such as an objection to the recruiters' appearance or mannerisms, negative views about the university, ${ }^{8,22}$ or distrust of the medical/research community. ${ }^{4,23,24} \mathrm{Re}-$ cruiter training exercises, including role-playing and shadowing of experienced recruiters, can help to minimize this variation, but it is likely that some will still exist between recruiters. Awareness of this inherent variability can prompt researchers to devise methods to monitor and evaluate the differences among recruiters and make adjustments to recruitment methods as necessary.

Our analysis has both methodological strengths and weaknesses. Strengths include the richness of the qualitative data and the detailed explanation of recruiter training methods. Limitations include the fairly low percentage of minority participants and the relative lack of diversity among the participating practices, both of which limit our ability to generalize our results. In addition, it is impossible to evaluate the representativeness of the participant group because of the lack of nonparticipant data.

\section{Conclusion}

Although participant recruitment remains challenging, our experience in SCOPE supports the importance of developing a standardized recruitment protocol, comprehensively training recruiters, and initiating regular recruitment meetings. Researchers are urged to publish their recruiter training methods and strategies for success so that others can learn from their experiences in a variety of recruitment scenarios.

We are indebted to all the participants who agreed to take part in the study, as well as the clinicians and staff members at each participating practice. We thank the New Jersey Primary Care Research Network, the SCOPE research team, and all of the SCOPE recruiters, especially Enid Cruz for her contributions during the recruitment debriefing session. Finally, we thank the National Cancer Institute for funding this research.

\section{References}

1. Amthauer H, Gaglio B, Glasgow RE, Dortch W, King DK. Lessons learned: patient strategies for a type 2 diabetes intervention in a primary care setting. Diabetes Educ 2003;29:673-81.
2. Ross S, Grant A, Counsell C, Gillespie W, Russell I, Prescott R. Barriers to participation in randomised controlled trials: a systematic review. J Clin Epidemiol 1999;52:1143-56.

3. Agras WS, Bradford RH. Recruitment: an introduction. Circulation 1982;66(Suppl IV):IV2-5.

4. Swanson G, Ward A. Recruiting minorities into clinical trials: toward a participant-friendly system. J Natl Cancer Inst 1995;87:1747-59.

5. Harris R, Dyson E. Recruitment of frail older people to research: lessons learnt through experience. J Adv Nurs 2001;36:643-51.

6. Smith SR, Jaffe DM, Petty M, Worthy V, Banks P, Strunk RC. Recruitment into a long-term pediatric asthma study during emergency department visits. J Asthma 2004;41:477-87.

7. Ory MG, Lipman PD, Karlen PL, et al. Recruitment of older participants in frailty/injury prevention studies. Prev Sci 2002;3:1-22.

8. Chang B-H, Hendricks AM, Slawsky MT, Locastro JS. Patient recruitment to a randomized clinical trial of behavioral therapy for chronic heart failure. BMC Med Res Methodol 2004;4:8.

9. Ellis PM. Attitudes towards and participation in randomised clinical trials in oncology: a review of the literature. Ann Oncol 2000;11:939-45.

10. Margitić S, Sevick MA, Miller M, et al. Challenges faced in recruiting patients from primary care practices into a physical activity intervention trial. Prev Med 1999;29:277-86.

11. Adams J, Silverman M, Musa D, Peele P. Recruiting older adults for clinical trials. Control Clin Trials 1997;18:14-26.

12. Petrovitch H, Byington R, Bailey G, et al. Systolic Hypertension in the Elderly Program (SHEP). Part 2: screening and recruitment. Hypertension 1991; 17(3 Suppl):II16-23.

13. Seltzer SE, Sullivan DC, Hillman BJ, Staab EV. Factors affecting patient enrollment in radiology clinical trials: a case study of the American College of Radiology Imaging Network. Acad Radiol 2002;9: 862-9.

14. Love MM, Pearce KA, Williamson MA, Barron MA, Shelton BJ. Patients, practices, and relationships: challenges and lessons learned from the Kentucky Ambulatory Network (KAN) CaRESS clinical trial. J Am Board Fam Med 2006;19:75-84.

15. Glickman SW, Anstrom KJ, Lin L, et al. Challenges in enrollment of minority, pediatric, and geriatric patients in emergency and acute care clinical research. Ann Emerg Med 2008;51:775-80.

16. Crabtree BF, Miller WL, Stange KC. Understanding practice from the ground up. J Fam Pract 2001; 50:881-7.

17. Stroebel CK, McDaniel RR Jr, Crabtree BF, Miller WL, Nutting PA, Stange KC. How complexity science can inform a reflective process for improvement 
in primary care practices. Jt Comm J Qual Patient Saf 2005;31:438-46.

18. Agrawal J, Syngal S. Colon cancer screening strategies. Curr Opin Gastroenterol 2005;21:59-63.

19. Klabunde CN, Frame PS, Meadow A, Jones E, Nadel M, Vernon SW. A national survey of primary care physicians' colorectal cancer screening recommendations and practices. Prev Med 2003;36:35262.

20. Bleiker EM, Menko FH, Taal BG, et al. Screening behavior of individuals at high risk for colorectal cancer. Gastroenterology 2005;128:280-7.

21. Rawl SM, Menon U, Champion VL, Foster JL, Skinner CS. Colorectal cancer screening beliefs. Cancer Pract 2000;8:32-7.

22. Rojavin MA, Downs P, Shetzline MA, Chilingerian R, Cohard-Radice M. Factors motivating dyspepsia patients to enter clinical research. Contemp Clin Trials 2006;27:103-11.

23. Corbie-Smith G, Thomas SB, St. George DMM. Distrust, race, and research. Arch Intern Med 2002; 162:2458-63.

24. Holcombe RF, Jacobson J, Li A, Moinpour CM. Inclusion of black Americans in oncology clinical trials: the Louisiana State University Medical Center experience. Am J Clin Oncol 1999;22:18-21.

\section{Appendix. Frequently Asked Questions (from Patients) for Supporting Colorectal Outcomes through Participatory Enhancements (SCOPE) Recruiter Training}

\section{Patient: Is there any test involved?}

Response(s): No, we only ask that you fill out this survey while you wait for your appointment.

2. Patient: Are you going to call my home or send me e-mails?

Response(s): No, we will not contact you at all. This is simply a one-time request for you to fill out a survey.

3. Patient: Can I do the survey without signing the consent form?

Response(s): No. Because we are with the University, we need to have your signature on the consent form. If someone at the University needed to check to see that you agreed to fill out the survey, we would have to show them the signed consent form. Otherwise, your information from the survey is kept completely confidential and no one will be able to identify you from your information.

4. Patient: Can I change my mind at any time?

Response(s): Yes, there is information in the consent form (which I'll give you a copy of) that explains what to do if you change your mind about participating.

5. Patient: I can't read or write so well. Can you do the questionnaire for me?

Response(s): [Response will depend on whether or not you can help them complete the survey privately.] If privacy can be assured: I can read the consent form and questions to you. Then you can tell me your answers and I'll mark them down. If you cannot ensure privacy: I'm sorry but there's not enough privacy for me to read the survey out loud to you. Thank you very much for offering to participate.

6. Patient: If my husband participates, can I write the answers for him because I know everything about him?

Response(s): That's fine but he will need to be the one who signs the consent form and he needs to be able to confirm that what you're writing down is accurate. [If the husband is called back into the examination room, it would not be acceptable for the wife to complete the survey on her own.]

7. Patient: I didn't have my colonoscopy (or other test) at this practice. Should I still fill this out?

Response(s): Yes, it is important for us to know if you've had this test even if it was done elsewhere.

8. Patient: I can't remember the date of my colonoscopy (or other) test. What should I do? Response(s): That's understandable. Please write down your best guess for this date.

9. Patient: Can I take the survey into the examination room with me?

Response(s): Yes, of course. You can use this clipboard to write on. I'll be here in the waiting room so when you're finished, please return the survey to me before you leave.

10. Patient: Can I take the survey home to finish it and then mail it back to you?

Response(s): No, we do require that you complete the survey here in the office and return it to me.

11. Patient: Did the doctor say it was okay to fill this out? Does the doctor know you're doing this?

Response(s): Yes, the physician(s) have given us permission to do this so that we can help the practice improve their cancer screening rates.

12. Patient: I took this last time. Can I do it again? Response(s): If you took it last year, then, yes you can fill it out again for us this year. [If the patient 
completed the survey this year, then they would not be able to do it again.]

13. Patient: How will filling out this survey benefit me?

Response(s): Unfortunately, we don't give anything directly to you, but your contribution (and that of the other patients who are also filling out surveys here) will help your doctors know how well they are doing in making sure patients are receiving appropriate cancer screening tests. So we really hope that our work with your practice will improve the patient care around cancer screening for all of the patients here.

14. Patient: Who will have access to my chart/ medical record?

Response(s): One of our research team members will review your chart on a one- time basis. No identifying information (eg, name, address, SSN, etc) will be taken. We assign you a number and all of your information is kept completely confidential. The information we do take from your chart (eg, height, weight, dates of any cancer screenings) is then entered in a computer file. This allows members of our research team to analyze the information from all of the patients in this practice to see how well your physicians are doing in making sure patients are receiving appropriate cancer screening tests. [You may refer the patient to page 2 of the consent form to note who else may see their infor- mation: Institutional Review Board, National Cancer Institute, etc.]

15. Patient: I don't have cancer, so will I be a good candidate?

Response(s): Yes, we would still like for you to participate because we want to know if patients who are eligible to receive certain cancer screening tests have received them. These tests are important tools for preventing cancer so it's important for us to know how well they are being used for any patient.

16. Patient: What if I don't know if I've had a test or not? What if I don't know all the answers? Response(s): That's OK. You can always ask me for help if something on the survey is unclear. If you don't know if you've had a test, you can indicate that on the survey. If you can't remember when you had a particular test, you can write your best guess.

17. Patient: Why do I have to sign the consent form if this is confidential?

Response(s): Keeping your health information private is very important to us and we have to follow University rules to do this study. One of the rules is that we must have proof that you agreed to fill out this survey for us-that's why we need your signature on the consent form. This form is kept separate in a locked cabinet from your survey information so we don't use your name at all. 